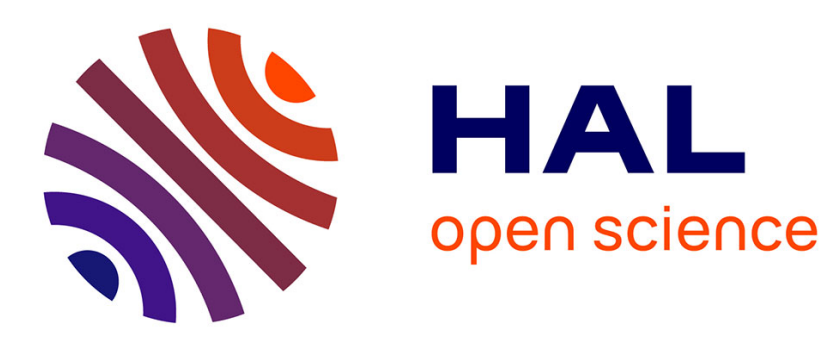

\title{
Acoustic scattering from simple and complex submerged objects
}

H. Überall

\section{To cite this version:}

H. Überall. Acoustic scattering from simple and complex submerged objects. Journal de Physique IV Proceedings, 1994, 04 (C5), pp.C5-779-C5-791. 10.1051/jp4:19945169 . jpa-00252849

\section{HAL Id: jpa-00252849 https://hal.science/jpa-00252849}

Submitted on 1 Jan 1994

HAL is a multi-disciplinary open access archive for the deposit and dissemination of scientific research documents, whether they are published or not. The documents may come from teaching and research institutions in France or abroad, or from public or private research centers.
L'archive ouverte pluridisciplinaire HAL, est destinée au dépôt et à la diffusion de documents scientifiques de niveau recherche, publiés ou non, émanant des établissements d'enseignement et de recherche français ou étrangers, des laboratoires publics ou privés. 


\title{
Acoustic scattering from simple and complex submerged objects
}

\author{
H. ÜBERALL
}

Department of Physics, Catholic University of America, Washington, DC 20064, U.S.A.

\begin{abstract}
The amplitude of sound scattered from submerged elastic objects is dominated by resonance phenomena corresponding to the mechanical eigenvibrations of the object. It is often difficult to predict the resonance frequencies of bounded elastic targets using exact methods. It has been shown, however, that resonances arise due to phase matching of circumferential waves traveling over the object surface. This leads to a standing-wave picture for the resonances, and a phase matching condition can be established that can lead to accurate predictions of resonances for objects of simple or complex shapes. In order to use this method, it is necessary to obtain the phase velocity of the surface waves. This is illustrated for spheroids and cylinders with hemispherical endcaps. The water loading of the object is of importance since it leads, in addition to the elastic surface waves, to the appearance of a fluid-borne "Scholte" or "Stoneley" wave which interacts with the lowest-order elastic wave, giving rise (for thin shells) to a repulsion phenomenon in the phase-velocity dispersion curves of the corresponding waves analogous to that known for energy levels in atomic or molecular systems. Of further importance is the effect of internal attachments to shell-type objects which also leads to the interaction of different wave types. For multilayered structures, it is finally shown that one may isolate and identify the resonances arising from individual layers using the method of Gérard.
\end{abstract}

\section{INTRODUCTION}

It is difficult to predict resonances for elongated elastic objects using exact elastodynamic theory [1,2], in particular if the object is not of canonical shape (sphere, infinite cylinder). It has been shown, however $[3,4]$ that in the scattering of sound from metallic objects, the dominant contribution to the scattering amplitude (besides that of the specular reflection) is provided by surface waves that propagate circumferentially over the object along geodesics, while internally transmitted waves (at least in the resonance region ka 10-100) have only small amplitudes [5]. Resonances arise from the surface wave 
contribution at those frequencies where the phase of the wave, after each circumnavigation of the object, comes back to the value it started out from ("phase matching").

The resonance frequencies are thus intimately connected with the phase velocity of the surface waves. Although the calculation of closed geodesics on objects of complex shapes is possible (but perhaps difficult [6]), approximations must be used in order to obtain the values of the phase velocities at each point of the geodesic. One model used by us previously is the "tangent sphere model" in which a sphere (on which the phase velocities are exactly calculable) is curvaturematched to a geodesic point in the propagation direction [4], with the effects of transverse curvature being small in general [7]. However, for the case of propagation along the cylindrical portion of an object, phase velocities of waves propagating in the axial direction can be obtained by calculation [8].

The phase matching condition, which is similar to the Bohr-Sommerfeld condition of atomic physics, is given by

$$
\oint \mathrm{ds} / \lambda_{\mathrm{sw}}=\mathrm{n}+1 / 2
$$

where $\lambda_{\text {sw }}$ is the wavelength of the surface wave. With $k_{\text {sw }}=\omega / v_{\text {sw }}\left(v_{\text {sw }}\right.$ being the phase velocity), this also reads

$$
\oint k_{\mathrm{sw}} \mathrm{ds}=2 \pi(\mathrm{n}+1 / 2) \text {. }
$$

The term $1 / 2$ added to the number $n$ of wavelengths spanning the closed path originates from the fact that at the two vertices of the bounded object, focal points arise at which a phase jump $\lambda / 2$ each takes place.

\section{THE PHASE MATCHING METHOD}

\section{$2 . I$ Experiments and Surface-Wave Interpretation for Elastic Finite- Length Cylinders}

The targets used in this part of our study are finite-length cylinders of diameter $\mathrm{D} \equiv 2 \mathrm{a}$; they are terminated by hemispheres and the overall length is $I$. Only axial or broadside incidence were considered here.

In order to solve Eq. (1b) for the complex resonance frequencies (the imaginary parts determining the resonance widths), the phase velocities of the surface waves must be known. For a steel sphere (such as at the endcaps) the complex eigenfrequencies can be found analytically [9], and are shown in Fig. 1. They are grouped into families, the lowest (labeled $\ell=1$ ) corresponding to the "Rayleigh" surface wave, the higher waves $(\ell=2,3 \ldots)$ being termed "Whispering Gallery waves". From Fig. 1 one obtains the phase velocities (at the discrete resonance points) by [9]

$$
\mathrm{v}_{\mathrm{sw}} / \mathrm{c}=\mathrm{ka} /(\mathrm{n}+1 / 2),
$$

$c$ and $k$ being sound speed and propagation constant in the ambient fluid. Figure 2 shows the $\ell=1$ phase velocities for a solid steel sphere (crosses) and, from a similar calculation, for a steel cylinder (solid curve) for the lowest-order wave propagating axially. These are the ingredients for the calculation of capped-cylinder resonances by phase matching, Eq. (1b). 
Results are shown in Fig. 3 for the corresponding Regge curves (real parts of the resonance frequencies plotted vs. mode number). The diamonds show the present predictions; crosses were previous predictions in which the cylinder dispersion curve of Fig. 2 had been approximated by its high-frequency constant "Rayleigh limit" which is shown to lead to disagreement at low frequencies. Asterisks are the results of a "bar wave approximation" [10] in which the cylinder was approximated by the model of an elastic bar; this is shown to lead to disagreement at higher frequencies. Finally, the circles present our experimental results (axial incidence) which confirm our present calculation and rule out the bar model.

For an $\mathrm{L} / \mathrm{D}=2$ solid tungsten carbide (WC) cylinder, high-resolution experimental results of the resonance spectrum obtained at the University of Le Havre [8,11] are shown in Fig. 4 for axial incidence, with the corresponding assignments $(n, l), n$ being the mode number (number of wavelengths spanning the meridian) and $\ell$ the type of the surface wave. From these results, one can extract the Regge curves of Fig. 5, showing as diamonds the present phase-matching predictions, (as crosses those using the Rayleigh limit as in Fig. 2), as asterisks the bar wave result, and as circles the experimental points. These are seen to confirm the present calculation in favor of the predictions that used the Rayleigh limit only.

\subsection{Elastic Spheroids}

Experiments on the scattering of short sound pulses from steel spheroids were carried out in a water tank. The incident signal was a 3-cycle pulse of $600 \mathrm{kHz}$. Aspect ratios of the spheroids were $a: b=1: 5,1: 2$ (prolate) and $2: 1,5: 1$ (oblate); $180^{\circ}$ backscattering was measured. Figure 6 shows a set of echo time series for various aspect angles $\beta$ on a $1: 2$ spheroid ( $\beta=0^{\circ} \ldots$ end-on, $\beta=90^{\circ} \ldots$ broadside incidence). The time series show an initial return pulse due to specular reflection, followed by a sequence of delayed pulses due to the radiation from circumnavigating surface-wave pulses (directly transmitted and internally reflected echoes are imperceptible small [5]).

The arrival times of the first surface wave pulses (arrows in Fig. 6) can be estimated theoretically if one knows the geodesics along which the surface waves propagate. In addition, one needs to know the group velocities of the surface waves which will mainly depend on the radius of curvature along the propagation direction. These group velocities can be estimated from those on a steel sphere, and it is found that they depend very little on the sphere radius (i.e., the radius of curvature) so that they could be assumed as nearly constant for our purposes. Under these assumptions, the measured arrival times of Fig. 7 (crosses for $1: 5$ spheroids, diamonds for $1: 2$ spheroids) are seen to be well described (solid lines) by our theory of propagation along geodesics.

\subsection{Phase Matching of Bending Resonances}

When considering bending or flexural resonances excited at oblique incidence on a spheroid, we can view the spheroid as a bar of variable radius. The resonance condition is here also that the flexural wave which has made a round trip is exactly in phase with its original starting value. This is the well-known condition for studying waves in any kind of linear resonator. 
Calculated flexural resonances of the water-loaded steel spheroid (of length $I$ and maximal diameter $D)$, using $T$-matrix theory are shown in Fig. 8 [12]. This reference also describes the analysis, based on Timoshenko's theory [13], of the resonances in terms of standing bar waves.

The analysis matches perfectly the dependence of the resonance frequencies on the aspect ratio L/D, which from Fig. 8 decrease with increasing value L/D. This appears unusual but is explained by the theory of bar waves.

\section{REPULSION OF PHASE VELOCITY CURVES}

\subsection{Infinite-Cylindrical Shells}

Calculations of acoustic scattering from infinite-cylindrical, airfilled metal shells at broadside incidence were perfomred in Ref. [14], and more recently in Ref. [15]. Using Eq. (2) (without 1/2, for the cylinder case) to obtain the phase velocities of the surface waves at the resonance values of ka. (the latter having been calculated from exact cylindrical-shell theory), the phase velocity dispersion curves of Fig. 9 were obtained, with the thickenss of the water-loaded steel shell being determined by the inner-to-outer radius ratio $\mathrm{b} / \mathrm{a}=0.8$.

These correspond to two waves, termed $A_{o+}$ and $A_{\circ-}$ whose dispersion curves repel each other at $\mathrm{ka} \sim 5$. Without fluid loading, however, one finds the dashed curve which corresponds to the Lamb wave $A_{0}$, and which follows $A_{o+}$ above the repulsion point but $A_{o-}$ below. The latter has the character of the fluid-borne scholte-stoneley wave, with phase velocity always below the sound velocity $c$ in water.

It could be shown [15] that although the $A_{o+}, A_{0-}$ curves are well separated, they don't each possess uniformly the same character. The portions of the curves that follow the dashed line represent the $A_{\circ}$ Lamb wave, and those that follow the horizontal dashed line at $c$ represent the Scholte-stoneley wave. This switch-over of the nature of the waves at a repulsion point has been known previously for the shift of atomic energy levels during molecular formation [16].

\subsection{Plates}

The same repulsion phenomenon can be demonstrated for the Lamb wave phase velocity dispersion curves of an infinite plate imbedded in a fluid. This is not so clearly visible e.g. for the example of an aluminum plate shown in Brekhovskikh's book [17]. However, if one reduces the shear speed to $C_{T}=2.0 \mathrm{~mm} / \mu \mathrm{s}$ (the compressional speed $c_{\mathrm{L}}$ being kept at its natural value of $6.4 \mathrm{~mm} / \mu \mathrm{s}$ ), the corresponding Fig. 10 [18] shows that the Lamb curves consist basically of two families: a steep family that approaches $c_{r}$, and that would solely be present if the plate supported shear waves only; and a flatter family that approaches $\mathrm{C}_{\mathrm{L}}$, and that would solely be present if the plate were fluid $\left(\mathrm{C}_{\mathrm{T}} \equiv 0\right)$. However, the actual curves repel each other near $\mathrm{c}_{\mathrm{L}}$, so that for a brief portion each of the steep curves turns "fluid", then becomes steep again.

\section{SHELLS WITH INTERNAL ATTACHMENTS}

Solutions to this problem have been pioneered by Guo [19], and by 
Klauson and Metsaveer [20].

Guo considered the scattering problem for an evacuated infinitecylindrical shell at normal sound incidence, the shell having a massspring system diametrically attached to it. The backscattered amplitude plotted vs. ka is shown in Fig. 11. While the empty shell amplitude is given by the dotted curve, the internally loaded shell (solid curve, at incidence angles $\theta_{0}=0^{\circ}$ parallel to the shell diameter along the massspring system, and at $45^{\circ}$ to it) shows an additional resonant fine structure due to the interaction between structural waves and the attachment; in fact the fine structure is not correlated to the resonance frequencies of either the empty shell or the internal system, but can be shown to be due to the excitation of flexural waves in the shell.

Similax observations apply to the example considered in Ref. [20], the infinite-cylindrical shell here being reinforced by a "stringer", i.e. two longitudinal plates attached normally to the shell interior in a diametrically opposite fashion. Figure 12 shows (dashed) the backscattering amplitude for the unreinforced shell, and (solid curve) the effect of the stringer.

The case of a cylindrical shell of finite length with a series of circular internal ribs spaced about uniformly was treated in Ref. [21]. The shell scattering was obtained by the Geometrical Theory of Diffraction, and the scattering from the ribs by curvature-corrected results of the theory for a flat plate carrying a rib. Figure 13 shows the target strength vs. frequency of a soft cylinder (radius $=10.5 \mathrm{in}$, length $=230$ in) with five times six ribs, at aspect angle $40^{\circ}$. Rib scattering effects are clearly visible, and can be predicted by the Bragg law both for the entire shell (short arrows), and for the six-ribs sub-assemblies (long arrows).

\section{RESONANCES OF MULTILAYER SYSTEMS}

Sound propagation in multilayer systems is usually treated by subjecting the solutions of the elastic wave equations to the boundary conditions at all interfaces, leading to possibly large algebraic equation systems without providing any physical insight, although the layer resonances [22] can be visualized in this way by numerical calculations. An ingenious approach by Gérard [23] replaces this procedure by consideration of the individual interfaces with appropriate reflection and transmission coefficients. This is combined with a Generalized Debye Series approach $[23,24]$ which describes the multiple reflections in the various layers. It permits, however, to isolate the resonances that originate from the multiple reflections in one given layer. In this way, the resonances (and possibly the inverse problem) of the multilayer system can be solved for each layer one-by-one [25].

\section{CONCLUSIONS}

This paper dealt with acoustic scattering from submerged elastic objects, and in particular with the sound-induced resonances. We showed that these can be predicted using phase matching of surface waves propagating along closed geodesics, in agreement with experimental results. For the case of spheroids, the arrival times following that of the specular echo confirm the surface wave propagation along geodesic paths. For the case of cylinders with hemispherical endcaps, very 
detailed agreement of phase matching predictions for the resonances with experimental results was obtained. For solid cylinders, the bar wave approximation was found to give good results at the lowest frequencies only; it does not fit at the higher frequencies where the surface wave model is appropriate.

The effect of fluid loading creates a Scholte-Stoneley surface wave in addition to the elastic waves; its dispersion curve undergoes (for thin shells) a repulsion phenomenon with that of the lowest elastic wave, with corresponding change of character of the wave. Shells with internal attachments show a fine structure of resonances compared to those of the empty shell. If the attachments are periodically spaced, Bragg-type interference effects can be shown to arise. For multilayer systems, individual-layer resonances can be identified using the generalized Debye series approach.

\section{REFERENCES}

[1] Werby, M. F., in Computational quantum physics (Umar, oberacker, Strayer and Bottcher, eds.) [American Institute of Physics Conference Proceedings 1992, vol. 260] pp. 180-206.

[2] Acoustic Resonance Scattering ( $H$. Überall, ed.) [Gordon and Breach, New York, 1992].

[3] Überall H., Dragonette L. R., and Flax, I., J. Acoust. Soc. Am. 61 (1977) 711.

[4] Überall H., Stoyanov Y. J., Nagl A., Werby M. F., Brown S. H., Dickey J. W., Numrich S. K., and D'Archangelo J. M., J. Acoust. SoC. Am. 81 (1987) 312 .

[5] Williams K. L., and Marston P. L., J. Acoust. Soc. Am. 78 (1985) 1093 .

[6] Merchant B. L., Nagl A., and Überall, H., IEEE Trans. Antennas Propagat. 37 (1989) 629.

[7] Franz W., and Klante K., IRE Trans. Antennas Propagat. AP-7 (1959) S68.

[8] Maze G., Décultot D., Lecroq F., Ripoche J., Bao X. L., and Überall H., J. Acoust. SOC. Am. (submitted).

[9] Werby M. F., Stoyanov Y. J., Dickey J. W., Keskin M. , D'Archangelo J. M., Nagl A., Stoyanov N. J., Castillo J. J., and Überall H., J. Acoust. 3 (1990) 201.

[10] Hackman R., Sammelmann G., Williams K., and Trivett D., J. Acoust. SOC. Am. 83 (1988) 1255.

[11] Maze G., Lecroq F., Décultot D., Ripoche J., Numrich S., and Überall H., J. Acoust. SoC. Am. 90 (1991) 3271.

[12] Werby M. F., and Überall H., Math Modeling and Sci. Computing, in 
press.

[13] Timoshenko S., Phil. Mag. 41 (1921) 744.

[14] Talmant M., Quentin G., Rousselot J. L., Subrahmanyam, J. V., and Überall H., J. Acoust. SoC. Am. 84 (1988) 681.

[15] Maze G., Léon F., Ripoche J., and Überall H., J. Acoust. Soc. Am. (submitted).

[16] See, e.g., Meijer P. H. E., and Bauer E., Group Theory [North Holland, Amsterdam 1965] p. 52.

[17] Brekhovskikh, L. M., Waves in Layered Media [Academic Press, New York, 1960].

[18] Überall H., Hosten B., Deschamps M., and Gérard A., J. Acoust. Soc. Am. (submitted).

[19] Guo Y. P., J. Acoust. SoC. Am. 91 (1992) 926.

[20] Klauson A., and Metsaveer J., J. Acoust. SoC. Am. 91 (1992) 1834.

[21] Moser P. J., Überall H., and Yuan J. R., J. Acoust. Soc. Am. (in press).

[22] Nagl A., Überall, H., and Hoover W. R., IEEE Trans. Geosc. Remote Sens. GE-20 (1982) 332.

[23] Gérard A., C. R. Acad. SCi. Paris 278 (1974) 1055.

[24] Gérard A., and Überall H., J. Acoust. SoC. Am. (to be published).

[25] Gérard A., and Überall H., J. Acoust. Soc. Am. (submitted). 


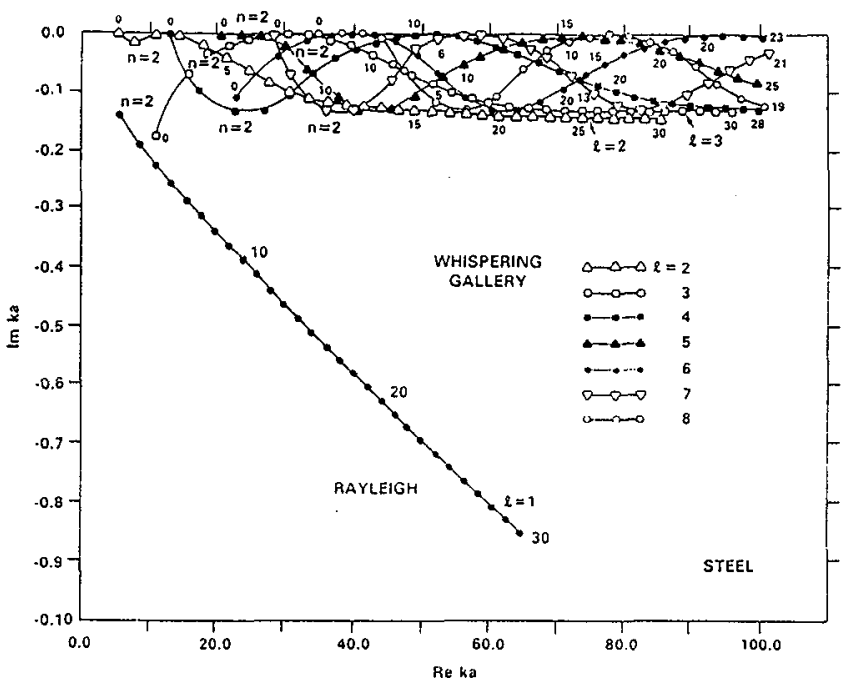

Fig. 1. Diagram of the resonance frequencies of a steel sphere in water, shown as poles of the scattering amplitude in the complex frequency (ka) plane.

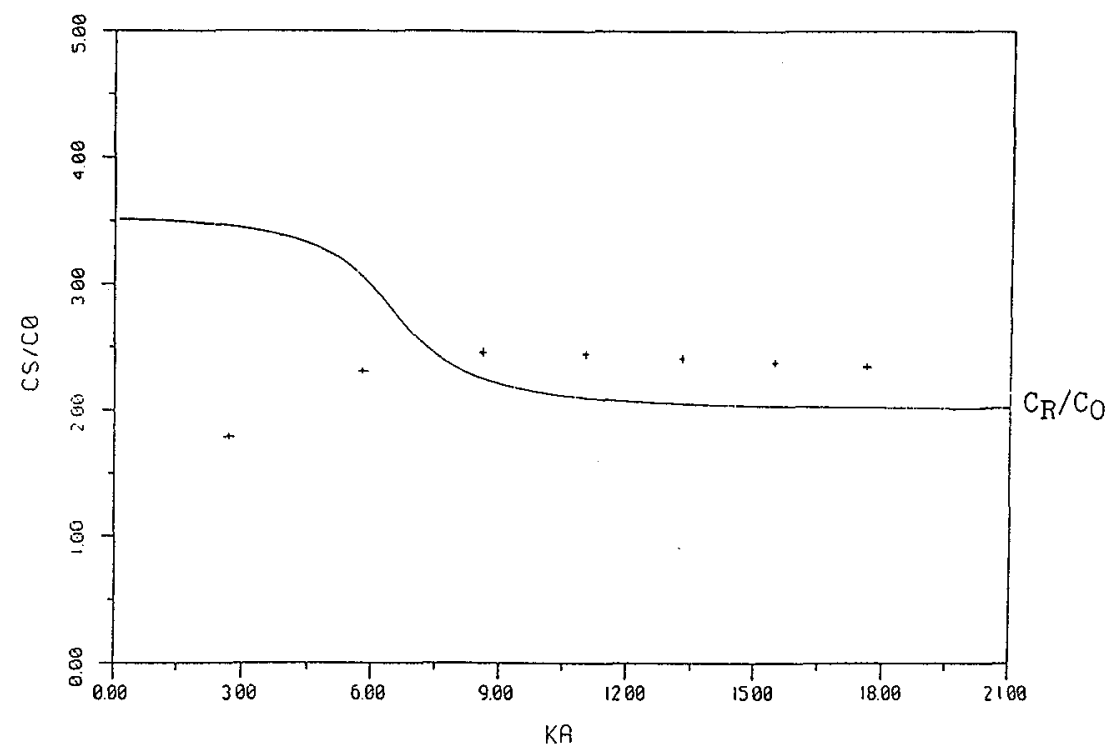

Fig. 2. Dispersion curves of the lowest-order axisymmetrical surface wave for an infinite solid steel cylinder (solid line), and lowest-order surface wave on a steel sphere (crosses). 


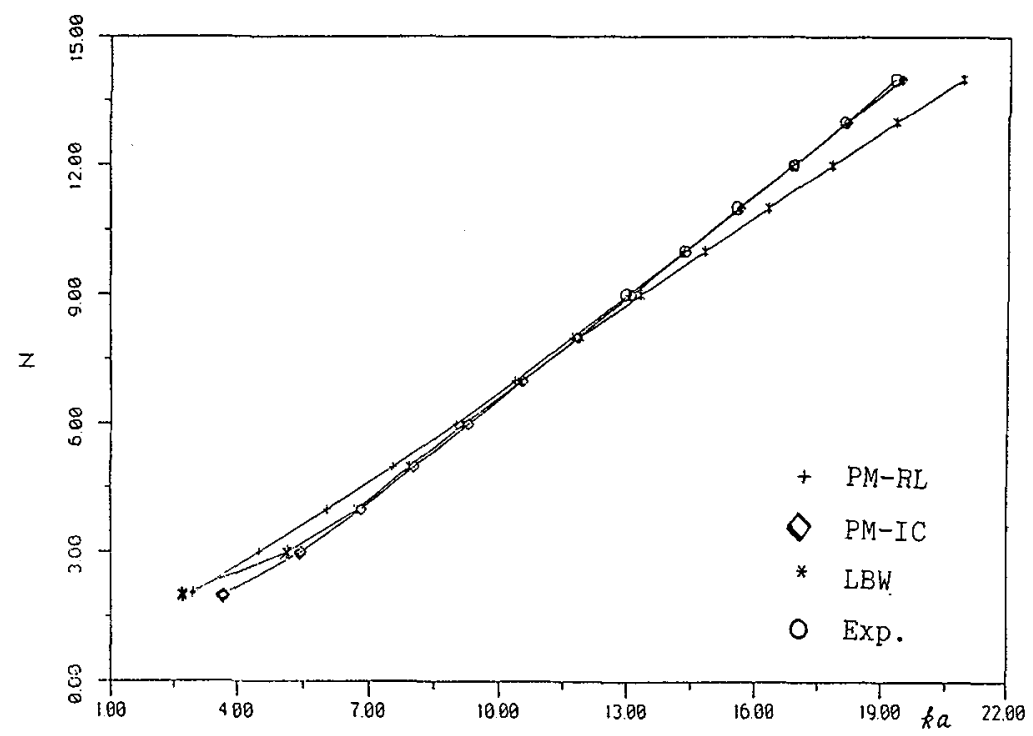

Fig. 3. Regge trajectories for solid steel cylinder with hemispherical endcaps, with aspect ratio $L / D=2$ for the case of end-on incidence.

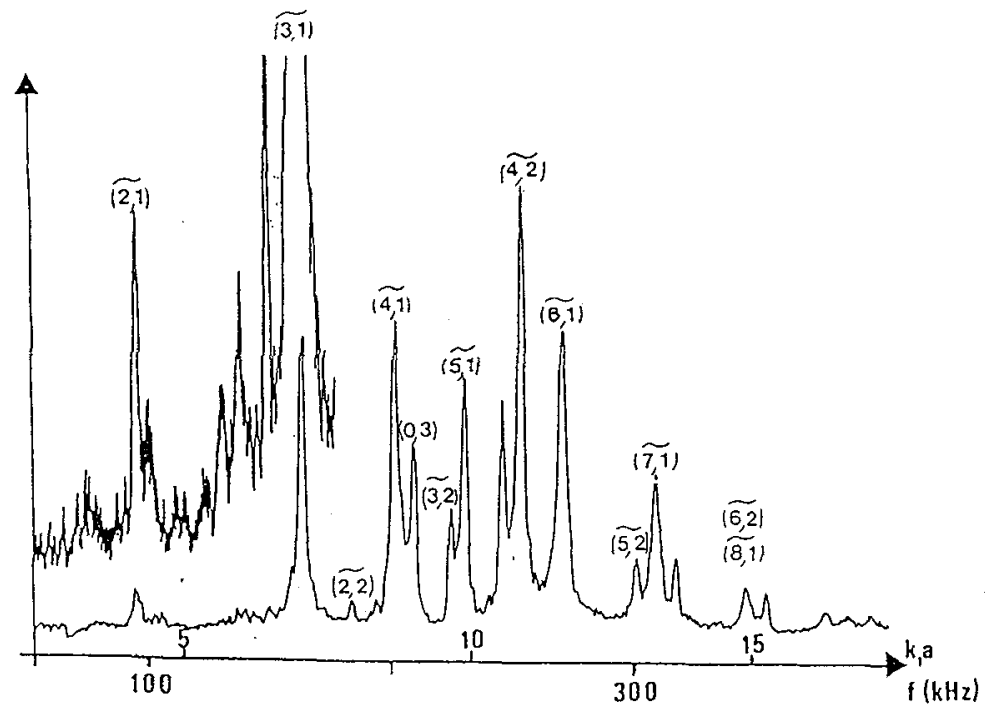

Fig. 4. Resonance response, from experiments at the University of Le Havre, of a solid WC cylinder with hemispherical endcaps, aspect ratio $\mathrm{L} / \mathrm{D}=2$, for the case of end-on incidence. 


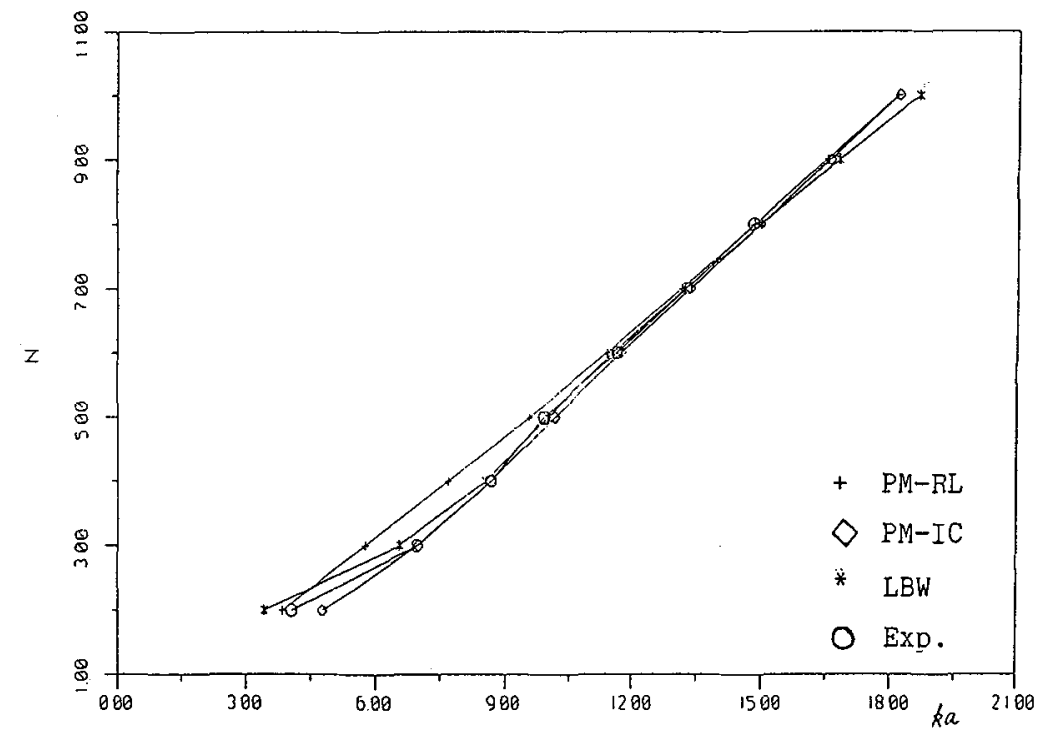

Fig. 5. Regge trajectories for solid WC cylinder with hemispherical endcaps, with aspect ratio $L / D=2$ for the case of end-on incidence.

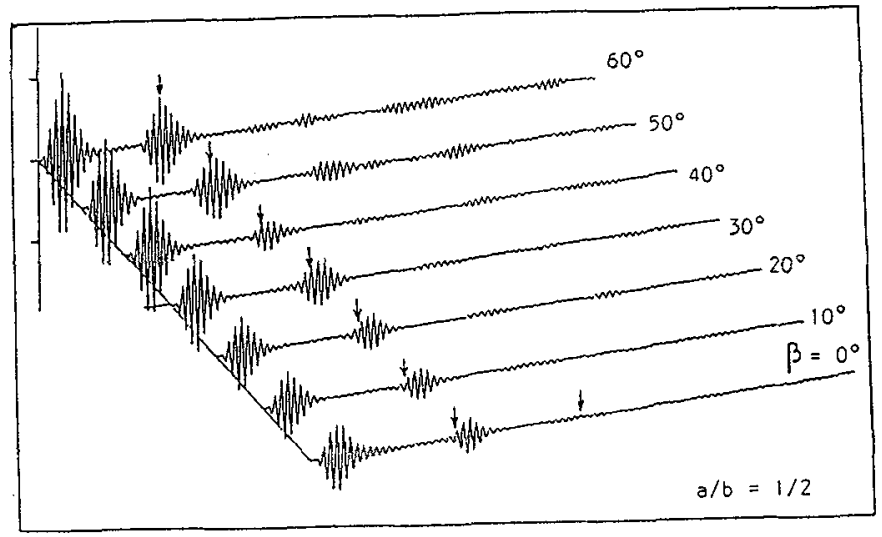

Fig. 6. Echo time series vs. incident angles for a $2: 1$ prolate steel spheroid. 


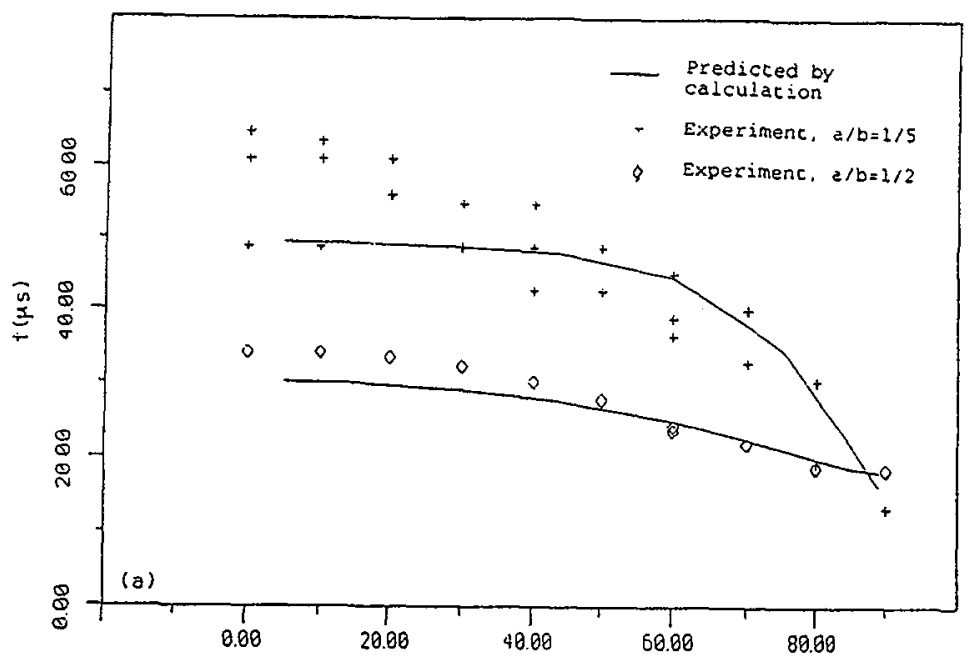

Fig. 7. Arrival time of the first elastic echo vs. incident angles, for $5: 1$ and $2: 1$ prolate steel spheroids.
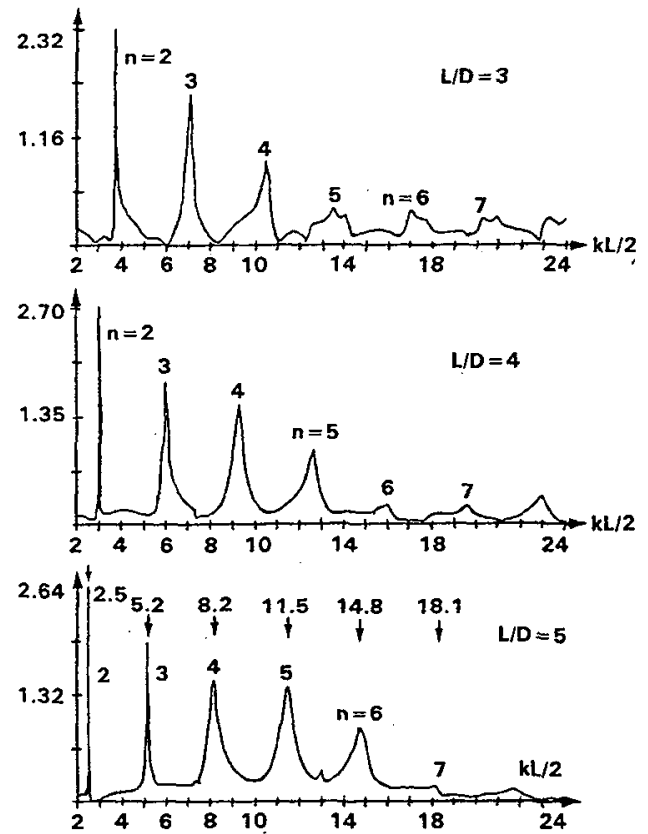

Fig. 8. Backscattered amplitude vs. frequency for a steel spheroid in water, at $45^{\circ}$ incidence. 


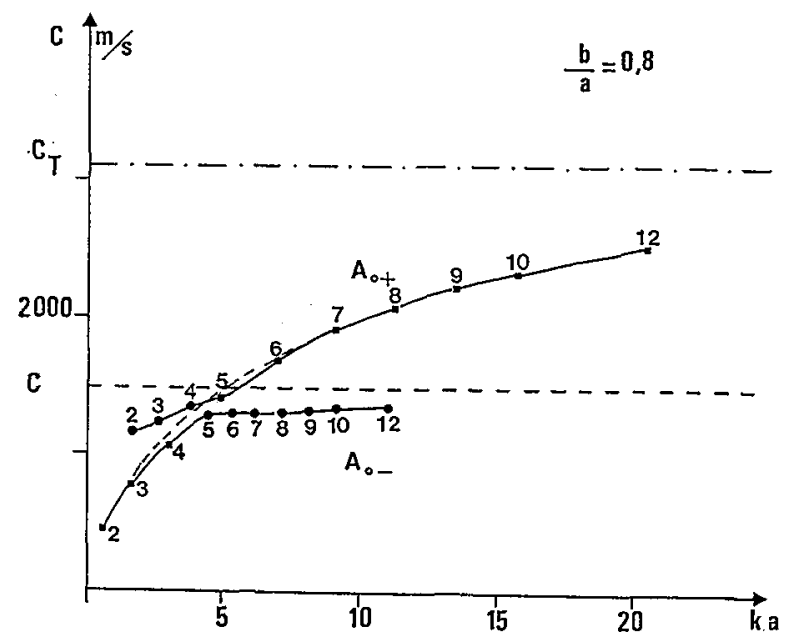

Fig. 9. Phase velocity dispersion curves for scholte-stoneley and $A_{\circ}$ Lamb waves on cylindrical steel shell in water, with $\mathrm{b} / \mathrm{a}=$ 0.8 .

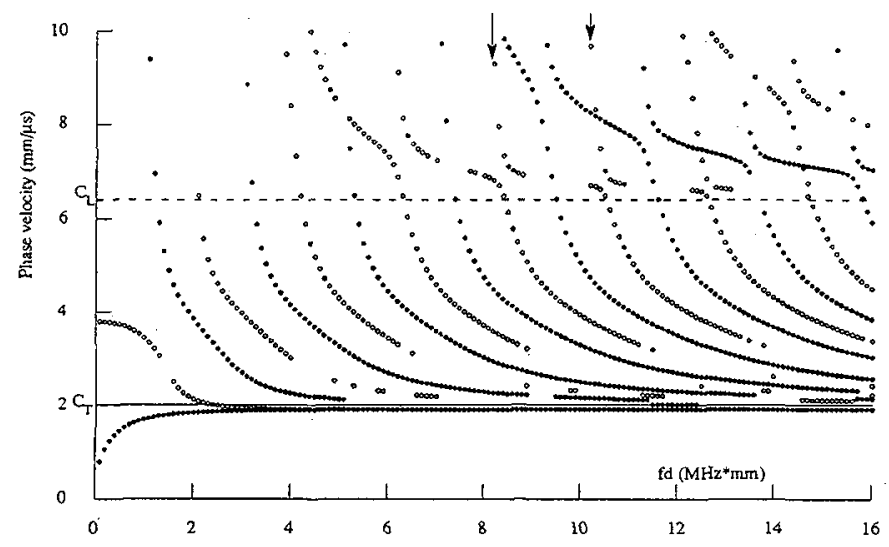

Fig. 10. Phase velocity dispersion curves on an "aluminum" plate in vacuum, with reduced shear speed $c_{T}=2 \mathrm{~mm} / \mu \mathrm{s}$. 


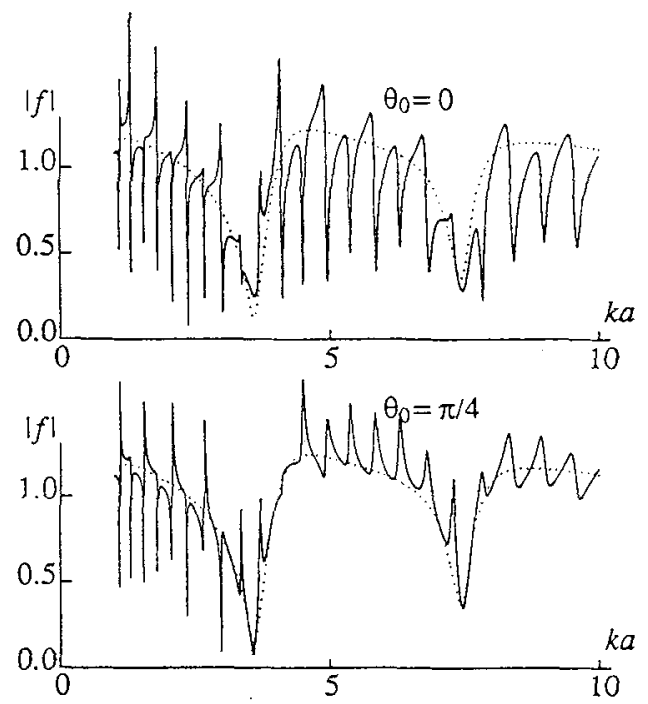

Fig. 11. Backscattered amplitude at different incident angles for a cylindrical steel shell of $1 \%$ thickness in water, with internally attached mass-spring system of $1 / 3$ the shell mass (solid curve); without internal loading (dotted).

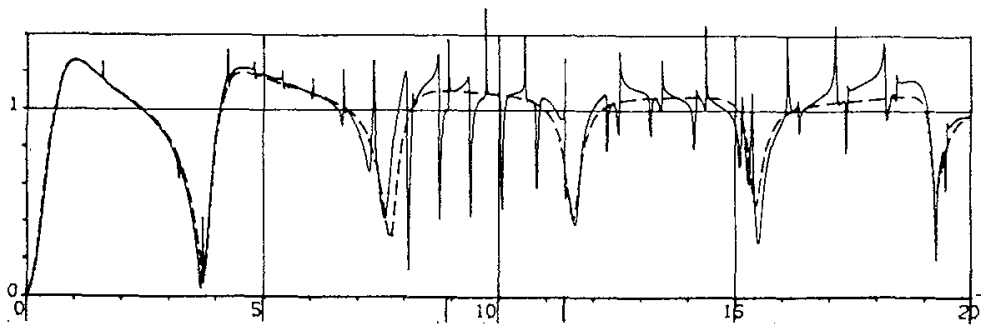

Fig. 12. Backscattered amplitude vs. frequency for a $2 \%$ thick aluminum shell in water, reinforced by a length-wise stringer (solid curve); without stringer (dashed).

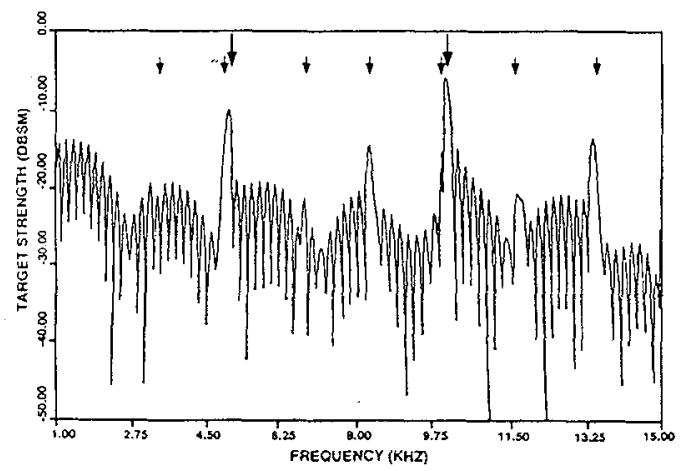

Fig. 13. Total target strength of a soft finite-length cylinder with 30 circular ribs vs. frequency at aspect angle $40^{\circ}$. Arrows indicate predicted Bragg interference maxima. 